\title{
An Effective Scheme for Pre-Emptive Priorities in Dual Bus Metropolitan Area Networks
}

\author{
Jörg Liebeherr ${ }^{*} \quad$ Ian F. Akyildiz ${ }^{* *} \quad$ Asser N. Tantawi ${ }^{* * *}$ \\ * Computer Science Division \\ University of California - Berkeley, \\ Berkeley, CA 94720, \\ U.S.A. \\ ** College of Computing, \\ *** IBM Research Division, \\ Georgia Institute of Technology, \\ Atlanta, GA 30332, \\ U. S. A.
Thomas J. Watson Research Center, Yorktown Heights, NY 10598, U. S. A

August 24, 1993

\begin{abstract}
The IEEE 802.6 standard for Metropolitan Area Networks does not provide multiple priority traffic for connectionless data services. A priority mechanism that was considered in earlier versions of the standard showed to be not effective. As of now, there exists no protocol for multiple access dual bus networks that is able to implement pre-emptive priorities and, at the same time, can satisfy minimal fairness requirements for transmissions at the highest priority level. In this study, a protocol with strictly pre-emptive priorities, i.e., a protocol that does not admit low priority traffic if the load from high priority traffic exceeds the capacity of the transmission channel, is presented. In addition, the protocol guarantees fairness for transmissions at the highest priority level. By introducing a general characterization of bandwidth allocation schemes for dual bus networks, existing priority mechanisms can be categorized according to the provided quality of service. The unique existence of a bandwidth allocation scheme for multiple priority traffic is shown with a full utilization of the channel capacity, with a fair distribution of bandwidth respective to traffic from a particular priority level and with pre-emptive priorities. The performance of the presented protocol is compared to existing proposals for multiple priority mechanisms. It is shown that adopting the new protocol results in shorter access delays for high priority transmissions. The protocol allows the stations of the network to react quickly to load changes. It is shown that the effectiveness of the priority scheme, compared to priority schemes using the bandwidth balancing mechanism, is less dependent on increasing the transmission speed of the network.
\end{abstract}

Key Words: Metropolitan Area Networks, Distributed Queue Dual Bus, Priorities, Media Access, Bandwidth Allocation 


\section{Introduction}

In July 1991, the Distributed Queue Dual Bus (DQDB) protocol was released as the IEEE 802.6 standard for Metropolitan Area Networks [11]. The standard left the protocol without an effective mechanism to support multiple priority traffic. Even though IEEE 802.6 supports the assignment of priorities, all connectionless data traffic must be sent at the lowest priority level ([11], p. 46). However, it is widely acknowledged that the support of multiple priority levels is needed to provide a variable quality of service to the stations of the network. Support of high priority traffic is especially needed for network control and management,

It is agreed upon that a satisfactory media access scheme for dual bus networks with multiple priority traffic should satisfy the following requirements [8]:

1. The bandwidth allocated to high priority traffic is independent from low priority traffic.

2. Within any priority level, the maximum bandwidth that can be allocated is equal for all stations.

3. Bandwidth is never wasted.

As of now, a priority mechanism is missing that satisfies all three requirements.

In this study we present a protocol for dual bus networks with multiple levels of priorities that satisfies all requirements listed above. We present a formal characterization for multiple-priority access schemes in dual bus networks. This allows us to present a unified view on bandwidth allocation schemes for dual bus networks. We are able to show the deficiencies of existing priority mechanisms. We show the unique solution to a bandwidth allocation scheme with a pre-emptive priority mechanism that does not waste bandwidth and satisfies fairness conditions for transmissions within each priority level. We develop a new protocol that is based on this unique solution. The new protocol uses results from [1] where we presented a uni-priority access protocol for dual bus networks that does not waste bandwidth and guarantees a fair distribution of bandwidth to the stations.

The paper is organized as follows. In section 2 we review the priority mechanism of the DQDB protocol. We discuss recent proposals that attempt to improve the priority mechanism of the DQDB protocol. In section 3 we categorize bandwidth allocation schemes for dual bus networks 
with multiple priorities, and derive a bandwidth allocation scheme that agrees with above mentioned requirements $1-3$. In section 4 we present a new protocol that implements the concept of a socalled strongly fair and waste-free bandwidth allocation with pre-emptive priorities. We compare the performance of our protocol with an implementation of a priority mechanism that satisfies above listed requirements 2 and 3 . We conclude our results in section 5 .

\section{Media Access Protocols for DQDB Networks with Multiple Priorities}

A $D Q D B$ network consists of two unidirectional buses with data flow in opposite directions. One bus is denoted by bus $\mathrm{A}$ and the other by bus B as shown in Figure 1. A slot generator at the

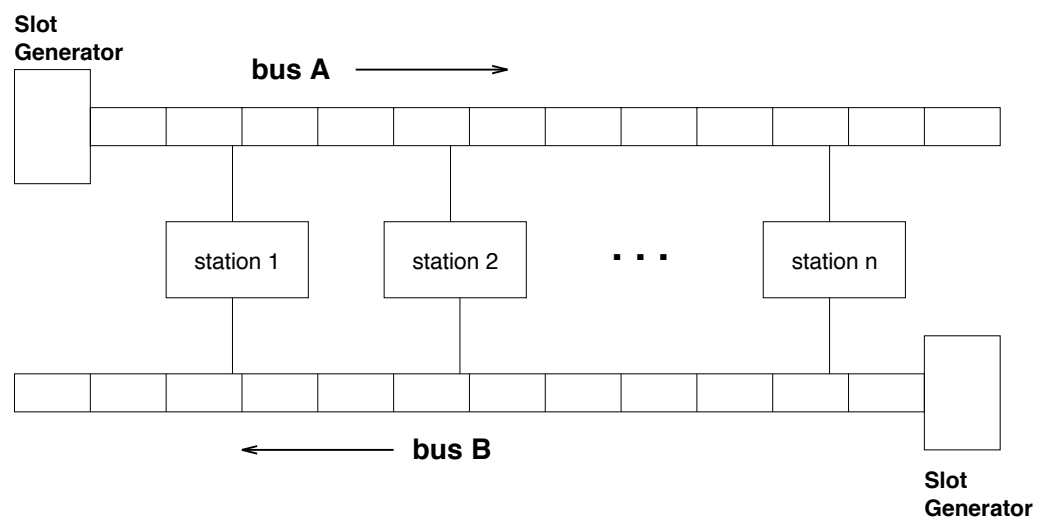

Figure 1: $D Q D B$ Network.

head of each bus emits empty fixed sized slots at a constant rate. Each station is connected to both buses. A station transmits data by filling in an empty slot on a particular bus. Note that due to the topology of the dual bus each station has to make a routing decision whether to use bus A or bus $\mathrm{B}$ for transmission dependent on the physical location of the destination station. Since the architecture of a dual bus network is symmetric we will focus on data transfer on bus A.

In subsection 2.1 we describe the media access protocol of the DQDB network with multiple priority levels that was used in early draft versions of the IEEE 802.6 standard [10]. In subsection 2.2 , we discuss proposals from the literature that attempt to enhance the priority mechanism in DQDB networks. 


\subsection{Media Access in a DQDB Network with Multiple Levels of Priorities}

The $D Q D B$ protocol prevents the stations close to the head of a bus from acquiring all empty slots by implementing a reservation scheme. A station having a segment ready for transmission on bus A notifies the stations closer to the the head of bus A by sending a reservation request on bus $B$.

In a DQDB network with $P$ priority levels, each slot contains $(P+1)$ access fields: a busy bit and $P$ request bits, one request bit for each priority level. A slot with the busy bit set indicates that the slot contains data. The request bit of priority $p$ set indicates a reservation request at priority level $p(1 \leq p \leq P)$. If a station writes data into an empty slot it sets the busy bit. A reservation request of priority $p$ is submitted by setting the priority- $p$ request bit.

For each priority level, a station keeps a queue of untransmitted segments. Only the segment at the head of a queue is allowed to submit a reservation request at the particular priority level. Note that setting the request bit may be delayed since a station has to wait for a slot which has the request bit not set. At each priority level, a station determines its turn to transmit a segment of priority $p$ with two counters, the request counter $\left(R Q_{p}\right)$ and the countdown counter $\left(C D_{p}\right)$ $(1 \leq p \leq P)$.

If a station does not have segments of priority level $p$ queved for transmission, it increments its $R Q_{p}$ counter for each passing slot on bus $\mathrm{B}$ having the request bit set at equal or higher priority levels $(q \geq p)$. It decrements $R Q_{p}$ for each empty slot the station detects on bus A. Upon arrival of a segment with priority $p$ to the station, $R Q_{p}$ is copied to $C D_{p}$ and then set to zero. Then $R Q_{p}$ is incremented for slots on bus $\mathrm{B}$ having the priority- $p$ request bit set. $C D_{p}$ is decremented for each empty slot passing by the station on bus A and incremented by one for each slot on bus B having the request bit set at higher priority levels $(q>p)$. When $C D_{p}$ reaches zero the segment of priority level $p$ is allowed to take the next empty slot for transmission.

The so-called bandwidth balancing mechanism [7] was included into the standard to achieve a fair distribution of bandwidth within a single priority class if the network is heavily loaded. The bandwidth balancing mechanism enforces at each priority level that a station uses only a fraction of the available bandwidth for transmissions. This is achieved by incrementing $R Q_{p}$ each time after a fixed number of $\beta^{1}$ transmissions of priority- $p$ segments.

\footnotetext{
${ }^{1}$ The default value is $\beta=8$.
} 


\subsection{Enhancements to the Priority Mechanism}

The priority mechanism as described in the previous subsection (including bandwidth balancing) is not effective. It was shown that it merely guarantees that stations with high priority traffic do not obtain more bandwidth than stations with low priority traffic $[5,15,16,18,19]$. Without the bandwidth balancing mechanism it is possible that stations with low priority traffic obtain more bandwidth than stations with high priority traffic [20].

Non-unity ratio bandwidth balancing [17] enforces that high priority traffic is assigned more bandwidth than low priority traffic by using different values of $\beta$ for traffic from different priority levels. Higher values for $\beta$ are used for high priority traffic. Note that high priority traffic is not independent from low priority traffic, i.e., increasing the amount of low priority traffic results in decreased traffic at higher priorities.

In symmetric bandwidth balancing [4], stations with low priority traffic leave an additional empty slot that is otherwise used for transmission each time after receiving a fixed number of high priority requests.

In [3], a priority mechanism with pre-emptive priorities is presented. Using additional bits in the slot header, stations notify each other about the highest priority level currently active on the network. A station refrains from transmitting if the highest active priority level is higher than the priority level of segments stored at the station. A fair distribution of bandwidth to stations transmitting at the highest priority level is not guaranteed.

In bandwidth balancing with global priority information [8], the slot header carries information on the priority level of transmitted data. The bandwidth balancing modulus $(\beta)$ is set equal for all priority levels. Under heavy load this priority mechanism distributes the bandwidth equally among transmissions at the same priority level. In addition, high priority traffic is independent from traffic at lower priorities. However, the scheme never utilizes the entire bandwidth of the bus. Variations of this scheme can be found in $[9,12,13]$. We refer to [14] for a detailed discussion of the literature on $D Q D B$.

As mentioned before, none of the above schemes achieves at the same time the independence of high priority traffic from low priority traffic, an equal maximum bandwidth allocation for stations transmitting at a particular priority level and a full utilization of the bandwidth of the dual bus. 


\section{Properties of Bandwidth Allocations with Multiple Levels of Priorities}

In this section we formally define properties of bandwidth allocation schemes for dual bus networks with multiple priorities. Because of the symmetry of the dual bus topology we only consider transmissions on one bus. Formally, a bandwidth allocation maps the traffic load from all stations into individual portions of the bandwidth that can be used for transmission.

Let $\mathcal{N}=\{1,2, \ldots, N\}$ be a set of stations and let $\mathcal{P}=\{1,2, \ldots, P\}$ be a set of priority levels. A high priority index denotes a high priority level. Let $\lambda_{i p}\left(\lambda_{i p} \geq 0\right)$ denote the load and $\gamma_{i p}\left(0 \leq \gamma_{i} \leq 1\right)$ denote the allocated bandwidth for station $i(1 \leq i \leq n)$ at priority level $p$ $(1 \leq p \leq P)$. Let the $(N \times P)$ matrices $\Lambda$ and $\Gamma$ be given by:

$$
\Lambda=\left(\begin{array}{cccc}
\lambda_{11} & \lambda_{12} & \cdots & \lambda_{1 P} \\
\lambda_{21} & \lambda_{22} & \cdots & \lambda_{2 P} \\
\vdots & \vdots & & \vdots \\
\lambda_{N 1} & \lambda_{N 2} & \cdots & \lambda_{N P}
\end{array}\right) \quad \Gamma=\left(\begin{array}{cccc}
\gamma_{11} & \gamma_{12} & \cdots & \gamma_{1 P} \\
\gamma_{21} & \gamma_{22} & \cdots & \gamma_{2 P} \\
\vdots & \vdots & & \vdots \\
\gamma_{N 1} & \gamma_{N 2} & \cdots & \gamma_{N P}
\end{array}\right)
$$

We define $\|\Gamma\|$ and $\|\Lambda\|$ as:

$$
\|\Lambda\|=\sum_{i=1}^{N} \sum_{p=1}^{P} \lambda_{i p} \quad \text { and } \quad\|\Gamma\|=\sum_{i=1}^{N} \sum_{p=1}^{P} \gamma_{i p} .
$$

Further we define $\Lambda_{p},\left\|\Lambda_{p}\right\|, \Gamma_{p}$ and $\left\|\Gamma_{p}\right\|(1 \leq p \leq P)$ as:

$$
\begin{aligned}
\Lambda_{p} & =\left(\begin{array}{c}
\lambda_{1 p} \\
\lambda_{2 p} \\
\vdots \\
\lambda_{N p}
\end{array}\right) & \text { and } & \left\|\Lambda_{p}\right\|=\sum_{i=1}^{N} \lambda_{i p} \\
\Gamma_{p} & =\left(\begin{array}{c}
\gamma_{1 p} \\
\gamma_{2 p} \\
\vdots \\
\gamma_{N p}
\end{array}\right) & \text { and } & \left\|\Gamma_{p}\right\|=\sum_{i=1}^{N} \gamma_{i p} .
\end{aligned}
$$

Definition 1 A bandwidth allocation for multiple priority levels is defined as a relation $\Omega \subseteq(\Lambda \times \Gamma)$ such that for all $i$ and $p(1 \leq i \leq N, 1 \leq p \leq P) \gamma_{i p} \leq \lambda_{i p}$ and $0 \leq\|\Gamma\| \leq 1$. 
Although relation $\Omega$ does not necessarily determine $\Gamma$ uniquely for given $\Lambda$ we will use the notation $\Gamma=\Omega(\Lambda)$. We denote the element in row $i$ and column $p$ of $\Omega(\Lambda)$ by $\Omega_{i p}(\Lambda)$. We denote the $p$ th column of $\Omega(\Lambda)$ by $\Omega_{p}(\Lambda)$. We use $\|\Omega(\Lambda)\|$ and $\left\|\Omega_{p}(\Lambda)\right\|$ to denote the sum of all elements in matrix $\Omega(\Lambda)$ and vector $\Omega_{p}(\Lambda)$, respectively.

We define the following fairness criteria for traffic of a particular priority level.

Definition $2 \Omega$ is fair if for all $\Lambda$

1. $(\forall p)(1 \leq p \leq P)(\forall i, j)(1 \leq i, j \leq N):\left(\lambda_{i p}<\lambda_{j p} \rightarrow \Omega_{j p}(\Lambda) \leq \Omega_{i p}(\Lambda)\right)$, and

2. $(\forall i, j)(1 \leq i, j \leq N):\left(\lambda_{i p}=\lambda_{j p} \rightarrow \Omega_{i p}(\Lambda)=\Omega_{j p}(\Lambda)\right)$.

The fairness conditions guarantee that within each priority level a station does not obtain more bandwidth than a station with a higher arrival rate, and stations with the same arrival rate obtain the same bandwidth.

Definition $3 \Omega$ is strongly fair if for all $\Lambda$

$(\forall p)(1 \leq p \leq P)\left(\exists \alpha_{p}^{*}>0\right)(\forall i)(1 \leq i \leq N):\left(\left(\lambda_{i p} \leq \alpha_{p}^{*} \rightarrow \Omega_{i p}(\Lambda)=\lambda_{i p}\right) \wedge\left(\lambda_{i p}>\alpha_{p}^{*} \rightarrow \Omega_{i p}(\Lambda)=\alpha_{p}^{*}\right)\right)$.

Strong fairness guarantees for priority level $p$ that stations with a load less than a threshold value $\alpha_{p}^{*}$ obtain all the bandwidth they need. All stations with a load exceeding the threshold value obtain the same bandwidth. Note that the condition for strong fairness implies fairness.

Next we formally describe bandwidth allocations that are able to utilize the entire bandwidth of the communication channel.

Definition $4 \Omega$ is waste-free if for all $\Lambda$

(i) If $\|\Lambda\|<1$, then $\|\Omega(\Lambda)\|=\|\Lambda\|$, and

(ii) If $\|\Lambda\| \geq 1$, then $\|\Omega(\Lambda)\|=1$.

If the traffic load from all stations is less than the capacity of the bus, a waste-free bandwidth allocation guarantees that all stations can transmit their load. If the traffic load exceeds the capacity, the entire bandwidth can be used for transmission, i.e., no bandwidth is wasted. 
The quality of service of the priority mechanism of a bandwidth allocation is categorized as follows.

Definition $5 \Omega$ implements pseudo-priorities if for all $\Lambda$

$$
(\forall p, q)(1 \leq p<q \leq P):\left(\left\|\Lambda_{q}\right\|>\left\|\Lambda_{p}\right\| \rightarrow\left\|\Omega_{q}(\Lambda)\right\| \geq\left\|\Omega_{p}(\Lambda)\right\|\right)
$$

Pseudo-priorities just guarantee that a station with high priority does not obtain less bandwidth than a station with low priority traffic.

Definition $6 \Omega$ implements weak priorities if for all $\Lambda$

$$
(\forall p, q)(1 \leq p<q \leq P):\left(\left\|\Lambda_{q}\right\|>\left\|\Lambda_{p}\right\| \rightarrow\left\|\Omega_{q}(\Lambda)\right\|>\left\|\Omega_{p}(\Lambda)\right\|\right)
$$

and

$$
(\forall p, q)(1 \leq q<p \leq P)(\exists \epsilon)(\epsilon>0):\left(\left\|\Omega_{p}(\Lambda)\right\|<\left\|\Omega_{p}\left(\begin{array}{cccccc}
\lambda_{11}+\epsilon & \cdots & \lambda_{1 q}+\epsilon & \lambda_{1 p} & \cdots & \lambda_{1 P} \\
\lambda_{21}+\epsilon & \cdots & \lambda_{2 q}+\epsilon & \lambda_{2 p} & \cdots & \lambda_{2 P} \\
\vdots & \vdots & & \vdots & & \vdots \\
\lambda_{N 1}+\epsilon & \cdots & \lambda_{N q}+\epsilon & \lambda_{N p} & \cdots & \lambda_{N P}
\end{array}\right)\right\|\right)
$$

Weak priorities guarantee that high priority traffic is allocated more bandwidth than low priority traffic. However, if the traffic load from low priority levels is increased by a constant $\epsilon$ the allocated bandwidth to the high priority stations is decreased. Therefore, weak priorities allow that stations with low priority traffic obtain a portion of the bandwidth even though the arrival rate of high priority traffic exceeds the total capacity of the bus.

Definition $7 \Omega$ implements strong priorities if for all $\Lambda$

$$
(\forall p, q)(1 \leq p<q \leq P):\left(\left\|\Lambda_{q}\right\|>\left\|\Lambda_{p}\right\| \rightarrow\left\|\Omega_{q}(\Lambda)\right\|>\left\|\Omega_{p}(\Lambda)\right\|\right)
$$

and

$$
(\forall p, q)(1 \leq q<p \leq P)(\forall \epsilon)(\epsilon>0):\left(\Omega_{p}(\Lambda)=\Omega_{p}\left(\begin{array}{cccccc}
\lambda_{11}+\epsilon & \cdots & \lambda_{1 q}+\epsilon & \lambda_{1 p} & \cdots & \lambda_{1 P} \\
\lambda_{21}+\epsilon & \cdots & \lambda_{2 q}+\epsilon & \lambda_{2 p} & \cdots & \lambda_{2 P} \\
\vdots & \vdots & & \vdots & & \vdots \\
\lambda_{N 1}+\epsilon & \cdots & \lambda_{N q}+\epsilon & \lambda_{N p} & \cdots & \lambda_{N P}
\end{array}\right)\right)
$$


Strong priorities assign more bandwidth to high priority traffic than to low priority traffic. In addition, the bandwidth allocated to high priority traffic is independent from the load of low priority traffic.

Definition $8 \Omega$ implements pre-emptive priorities if for all $\Lambda$

$$
(\forall p)(1 \leq p \leq P):\left(\sum_{q>p}\left\|\Lambda_{q}\right\|>1 \rightarrow\left\|\Omega_{p}(\Lambda)\right\|=0\right)
$$

An allocation with pre-emptive priorities does not admit low priority traffic if the traffic demand from higher priorities exceeds the total bandwidth. Note that strong priorities imply weak priorities, and weak priorities imply pseudo-priorities. In addition, we can follow from Definitions 4 and 7 that a waste-free bandwidth allocation $\Omega$ with strong priorities implements pre-emptive priorities, and pre-emptive priorities require a waste-free allocation with strong priorities. In [1] we showed the following properties for dual bus networks with a single level of priorities:

Lemma 1 Uni-priority DQDB without bandwidth balancing [10] is waste-free, but not fair.

Lemma 2 Uni-priority DQDB with bandwidth balancing [11] is strongly fair, but not waste-free.

With definitions $5-8$ we can show the following properties of priority mechanisms for dual bus networks:

Lemma 3 (i) The priority mechanism of IEEE 802.6/D12 [10] implements pseudo-priorities.

(ii) The priority mechanism of non-unity ratio bandwidth balancing [1 7$]$ allows to implement weak but not strong priorities.

(iii) The priority mechanism of DQDB with bandwidth balancing and global priority information [8] has strong priorities, but not pre-emptive since the bandwidth allocation is not waste-free.

\section{Proof:}

(i) Since the bandwidth balancing mechanism ensures strong fairness [1], it clearly guarantees pseudo-priorities. To show that the conditions for weak priorities are not satisfied assume a 
network where each station transmits at one priority level and each station is heavily loaded. Then the allocated bandwidth to a station $i$ transmitting with priority $p$ is given by [8]:

$$
\Omega_{i p}(\Lambda)=\frac{\beta}{1+\sum_{r=1}^{P} \beta \cdot K_{r}}
$$

where $K_{r}$ denotes the number of stations transmitting at priority level $r$. Since $\Omega_{i p}(\Lambda)=$ $\Omega_{j q}(\Lambda)$ (for $1 \leq i, j \leq N, 1 \leq p, q \leq P$ ) regardless of the load at different priority levels, the condition for weak fairness is clearly violated.

(ii) We consider the heavy load scenario as given above. In non-unity ratio bandwidth balancing we have different values for the modulus at each priority level, with $\beta_{p}>\beta_{q}$ if $p>q$. The bandwidth allocated to a station is calculated by [17]:

$$
\Omega_{i p}(\Lambda)=\frac{\beta_{p}}{1+\sum_{r=1}^{P} \beta_{r} \cdot K_{r}}
$$

Then, $\Omega_{i p}(\Lambda)>\Omega_{j q}(\Lambda)$ if $p>q$. However, strong priorities are not satisfied, since the bandwidth allocation to stations with high priorities is dependent on low priority traffic.

(iii) Again we consider the heavy load scenario. The allocated bandwidth to station $i$ with priority$p$ traffic is given by [8]:

$$
\Omega_{i p}(\Lambda)=\frac{\beta}{\prod_{q=p}^{P}\left(1+\beta \cdot K_{q}\right)}
$$

Weak priorities are satisfied since $\prod_{q=p}^{P}\left(1+\beta_{q} \cdot K_{q}\right)<\prod_{q=p^{\prime}}^{P}\left(1+\beta \cdot K_{q}\right)$ for $p>p^{\prime}$. The conditions for strong priorities are satisfied since the allocated bandwidth $\Omega_{i p}$ is independent from priority- $q$ traffic with $q<p$ (equation (3)). To show that the allocation is not waste free we simply verify that:

$$
\sum_{p=1}^{P} \sum_{i=1}^{K_{p}} \frac{\beta}{\prod_{q=p}^{P}\left(1+\beta \cdot K_{q}\right)}<1
$$

An ideal bandwidth allocation with multiple priority traffic combines strong fairness, waste-freedom and strong priorities. The following theorem states that such an allocation can be found.

Theorem 1 There exists exactly one bandwidth allocation $\Omega^{*}$ that is strongly fair, waste-free and has strong priorities for all $\Lambda . \Omega^{*}$ is determined by the unique solution to the following system of 
equations:

$$
\begin{gathered}
\Omega_{i p}^{*}(\Lambda)=\left\{\begin{aligned}
& 0 \text { if }(\exists q)(q>p):\left(\left|\mathbf{O}_{q}\right|>0\right) \\
& \min \left\{\lambda_{i p}, \frac{\sum_{q \geq p, j \in \mathbf{U}_{q}} \lambda_{j q}}{\left|\mathbf{O}_{p}\right|}\right\} \quad \text { otherwise }
\end{aligned}\right. \\
\mathbf{U}_{p}=\left\{j \mid \lambda_{j p}=\Omega_{i p}^{*}(\Lambda)\right\} \\
\mathbf{O}_{p}=\left\{j \mid \lambda_{j p}>\Omega_{i p}^{*}(\Lambda)\right\} \\
(1 \leq i \leq N, 1 \leq p \leq P)
\end{gathered}
$$

$\mathbf{U}_{p}$ denotes the set of underloaded stations with priority- $p$ traffic, i.e., stations which can satisfy their bandwidth demand of priority- $p$ traffic. $\mathbf{O}_{p}$ denotes the set of overloaded stations with priority- $p$ traffic, i.e., stations with a load at priority level $p$ exceeding the allocated bandwidth. Note that no bandwidth is allocated to a station if the set of overloaded stations at higher priority levels is nonempty.

Proof: The proof is given in Appendix A.

In the remaining part of this section, we show that the strongly fair and waste-free bandwidth allocation with strong priorities can be obtained in a distributed way. Recall that a waste-free bandwidth allocation with strong priorities is pre-emptive.

Definition 9 For each $(i, p)(1 \leq i \leq N, 1 \leq p \leq P)$ define $\mathbf{O}_{p}^{(>i)}$ by:

$$
\mathbf{O}_{p}^{(>i)}=\left\{j \mid j \in \mathbf{O}_{p} \wedge i<j \leq N\right\}
$$

$\mathbf{O}_{p}^{(>i)}$ denotes the set of stations in the overload set of priority level $p$ with higher index than $i$.

Theorem 2 Given a strongly fair and waste-free bandwidth allocation $\Omega^{*}$ with strong priorities. Then $i \in \mathbf{O}_{p}$ if and only if

$$
\lambda_{i p}>\delta_{i p}
$$

with

$$
\delta_{i p}= \begin{cases}0 & \text { if }(\exists q)(q>p):\left(\left|\mathbf{O}_{q}^{(>i)}\right|>0\right) \\ \frac{1-\sum_{q \geq p, j<i} \Omega_{j q}^{*}(\Lambda)-\sum_{q>p} \Omega_{i q}^{*}(\Lambda)-\sum_{q \geq p, j>i, j \in \mathbf{U}_{q}} \lambda_{j q}}{\left|\mathbf{O}_{p}^{(>i)}\right|+1} & \text { otherwise }\end{cases}
$$


Proof: The proof is given in Appendix B.

From the proof of Theorem 2 we additionally obtain:

Corollary 1 Given a strongly fair and waste-free bandwidth allocation with strong priorities. For all $i \in \mathbf{O}_{p}$

$$
\Omega_{i p}^{*}(\Lambda)=\frac{1-\sum_{q \geq p, j>i, j \in \mathbf{U}_{q}} \lambda_{j q}-\sum_{q>p} \Omega_{i q}^{*}(\Lambda)-\sum_{q \geq p, j<i} \Omega_{j q}^{*}(\Lambda)}{\left|\mathbf{O}_{p}^{(>i)}\right|+1}
$$

Corollary 2 The strongly fair and waste-free bandwidth allocation with strong priorities can be implemented if each station $i$ with $\lambda_{i p}>0$ knows the following set of parameters:

$$
\left(\left|\mathbf{O}_{p}^{(>i)}\right|, \sum_{j>i, q \geq p, j \in \mathbf{U}_{q}} \lambda_{j q}, \sum_{q>p} \Omega_{i q}^{*}(\Lambda), \sum_{j<i, q \geq p} \Omega_{j q}^{*}(\Lambda)\right) .
$$

In the following section we present a media access protocol for dual bus networks with multiple priority levels that uses the results from Theorem 2 and Corollaries 1 and 2.

\section{A Strongly Fair and Waste-Free Multiple Access Protocol with Pre-Emptive Priorities}

We only consider data transmission on bus A. We will use the station index to denote the relative physical distance to the slot generator of bus A. So station 1 will denote the station closest to the slot generator of bus A, station 2 the second closest station, etc. . The stations with greater index than station $i$ are referred to as the downstream stations of station $i$, stations with smaller index are referred to as the upstream stations.

\subsection{Design Concepts}

Transmission of traffic from a station is handled independently for each priority level. Each station consists of so-called modules that control the transmission of traffic from a particular priority level. The modules of a station are organized such that modules for high priority traffic are upstream from the modules for low priorities (Figure 2). We denote the module that controls traffic of 


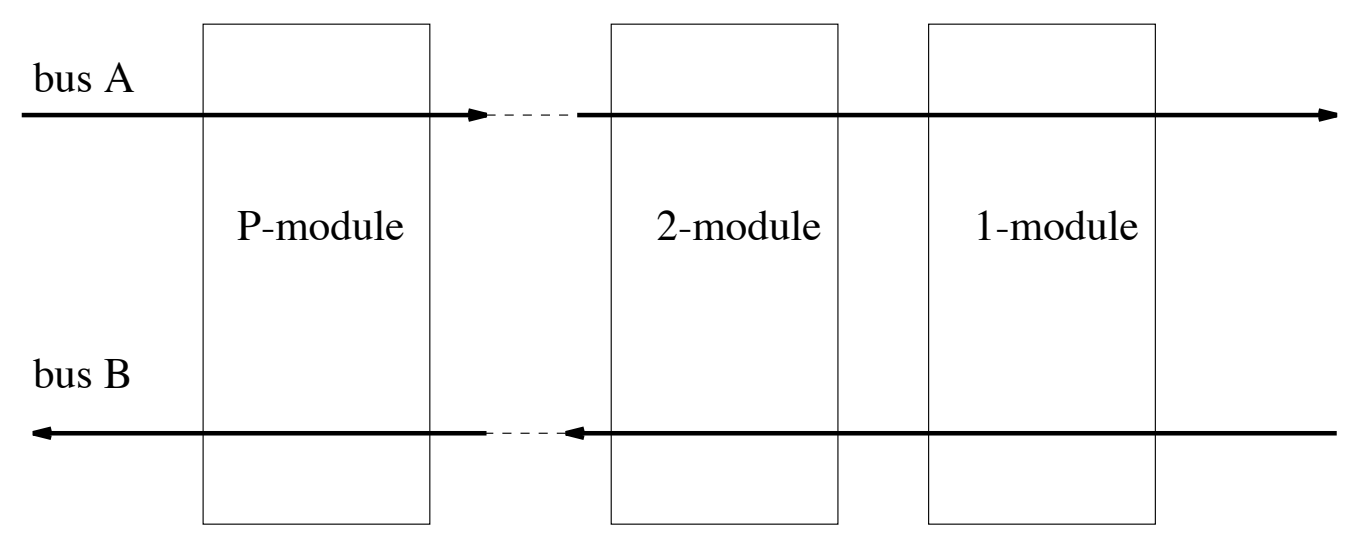

Figure 2: Priority Modules of a Station.

priority level $p$ as the $p$-module. Each module is considered as either underloaded or overloaded. An underloaded $p$-module can satisfy its bandwidth requirements at priority $p$, an overloaded $p$-module is characterized by an offered load that exceeds the allocated bandwidth. Both underloaded and overloaded modules use bus $B$ to send reservation requests to upstream stations. Underloaded $p$-modules send a priority- $p$ reservation request for each segment. If an underloaded $p$-module becomes overloaded, it stops sending reservation requests, and sends a signal on bus B to notify the upstream stations that it is overloaded. Once the signal is sent, no more reservations requests are submitted. If an overloaded $p$-module becomes underloaded, it sends a signal on bus B to indicate to upstream stations that it became underloaded. Then, the $p$-module resumes sending priority- $p$ reservation requests, one for each segment of priority $p$.

Before a $p$-module is allowed to transmit a segment of priority $p$, it has to consider all reservations from downstream modules with equal or higher priority. For each reservation request of priority $\geq p$ and for each overloaded module of priority $p$ downstream, the station has to leave an empty slot on bus A. If a station receives an overload signal of priority $>p$, it ceases transmission until the opposite signal is received.

From Theorem 2 we know the necessary and sufficient overload conditions for the $p$-module of station $i$. Since we use the station index to denote the relative position of a station and since the modules of a station are ordered as given in Figure 2, the values in equation (10) can be calculated by:

$\lambda_{i p} \quad: \quad$ load of priority-p-traffic to $p$-module 
of station $i$,

$\sum_{q \geq p, j<i} \Omega_{j q}^{*}(\Lambda)+$

$\sum_{q>p} \Omega_{i q}^{*}(\Lambda) \quad: \quad$ rate of busy slots on bus $\mathrm{A}$ from priorities $\geq p$

seen by $p$-module of station $i$,

$\sum_{q \geq p, j>i, j \in \mathbf{U}_{q}} \lambda_{j q}:$ rate of reservations requests on bus $\mathrm{B}$ of priorities

$\geq p$ received by $p$-module of station $i$,

$\left|\mathbf{O}_{p}^{(>i)}\right| \quad:$ number of overloaded $p$-modules downstream on bus A.

\subsection{Implementation}

Each slot carries the following bits in the slot header: one request bit and one busy bit for each priority level, a plus bit and a minus bit. For three priorities $(P=3)$, the access control (ACF) field of the slot header has a structure as shown in Figure $3^{2}$. The $p$ th busy bit is set by a module of priority level $p$ when inserting a segment of priority $p$ into a slot.

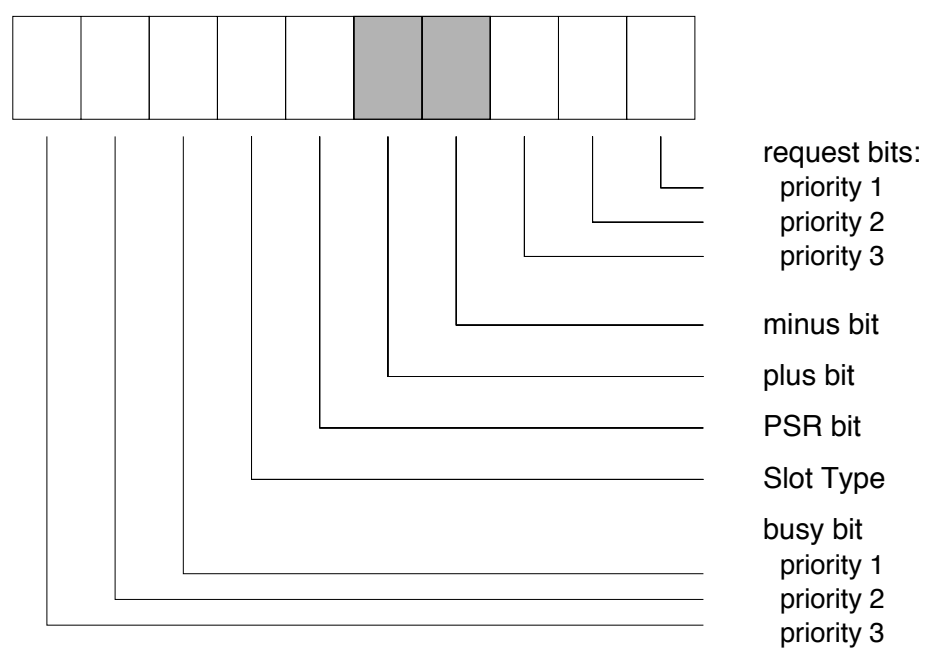

Figure 3: Access Control Field.

Each underloaded $p$-module sends one reservation request for the segment on top of the queue of untransmitted priority- $p$ segments. This is done by setting the request bit of priority $p$ in a slot on bus B. If an underloaded module becomes overloaded, it sets a plus bit and a priority- $p$ request

\footnotetext{
${ }^{2}$ The slot type bit and the PSR bit are described the IEEE 802.6 standard [11].
} 
bit in a slot on bus B. After setting the plus bit, no more reservation requests are transmitted. If an overloaded $p$-module becomes underloaded, it sets a minus bit and a request bit of priority $p$ in a slot of bus $B$, and resumes setting priority- $p$ request bits, one for each segment. Note that neither plus nor minus bits can be set in slots that have the request bit set at any priority level. Note however, that request bits in a slot can be set at all priority levels.

A $p$-module determines its turn to transmit a (priority- $p$ ) segment with five counters, the request counter $(R Q)$, the countdown counter $(C D)$, the overload request counter $(O R Q)$, the overload countdown counter $(O C D)$ and the stop-transmission counter (STOP). An idle $p$-module, i.e., a $p$-module that does not have a priority- $p$ segment queued for transmission, increments $R Q$ for each passing slot on bus $\mathrm{B}$ with the request bit set at priority level $p$ or higher. $O R Q$ is incremented when a slot on bus $\mathrm{B}$ passes by with both the plus bit and the priority- $p$ request bit set. ORQ is decremented by one for each slot with both the minus bit and the priority-p request bit set (If $O R Q=0$ then $O C D$ is decremented). STOP is incremented by one if a slot passes by with both the plus bit set and a request bit at a priority $>p$ set, and $S T O P$ is decremented by one for each slot with both the minus bit and the request bit at a priority $>p$ set.

At an idle $p$-module, $R Q$ is decremented for each empty slot and for each busy slot with the busy bit set at priority $<p$ that passes by on bus $\mathrm{A}$ ( $R Q$ is not decremented if $R Q=0$ ).

When a priority- $p$ segment arrives at an idle module, the contents of $R Q$ and $O R Q$ are copied to $C D$ and $O C D$, respectively, and both $R Q$ and $O R Q$ are set to zero. Now, $R Q$ is incremented for each slot with set request bit at priority $p$ on bus B. $C D$ is incremented for each slot on bus B with the request bit set at priority $>p$. ORQ is incremented for each slot on bus $\mathrm{B}$ with the plus bit and the priority- $p$ request bit set, and $O R Q$ is decremented for each slot on bus $\mathrm{B}$ with both the minus bit set and the priority- $p$ request bit set. If a minus bit and priority- $p$ request bit are read on bus B, when $O R Q$ is zero, $O C D$ is decremented by one. For each empty slot and for each busy slot of priority $<p$ on bus $\mathrm{A}, C D$ is decremented by one. If $C D$ is zero the module decrements $O C D$ by one, and, at the same time, increments $O R Q$ by one. If an empty slot arrives at the $p$-module and $C D, O C D$ and $S T O P$ are zero, the empty slot is used for transmission of the segment. If the $p$-module has more segments waiting for transmission, $R Q$ and $O R Q$ are copied to $C D$ and $O C D$, and then set to zero.

With Theorem 2, each $p$-module can determine by itself whether it is overloaded or underloaded. 
The rates needed to calculate equation (9) are obtained from the values of counters. Most of the required information is already stored in counters $R Q, C D, O R Q, O C D$, and $S T O P$. Three additional counters are needed. NoSeg contains the total number of segments (of priority $p$ ) queued for transmission at the $p$-module, SlotCtr is incremented for each arriving slot on bus B, and Bsy is incremented for each busy slot read on bus A with priority $p$ or higher. A p-module determines its state each time Basis ${ }^{3}$ slots have passed by on bus B (SlotCtr = Basis). Then it calculates:

$$
\text { Quota }= \begin{cases}0 & \text { if } S T O P>0 \\ \frac{B a s i s-B s y-R Q-C D}{O R Q+O C D+1}, & \text { otherwise }\end{cases}
$$

and sets counters SlotCtr and Bsy to zero. Quota provides the maximum number of slots a module is allowed to transmit during a period of Basis slots. If NoSeg $>$ Quota, the p-module is overloaded, otherwise the $p$-module is underloaded.

\subsection{Evaluation}

In order to show that our protocol achieves the objectives of strong fairness, waste-freedom and pre-emptive priorities, we execute simulation runs of file transfer scenarios ${ }^{4}$. We compare our protocol with the priority scheme presented in [8] to our knowledge is the only (verified) bandwidth allocation scheme that satisfies the conditions for both strong priorities and strong fairness.

We study a dual bus network with four stations that start file transfers on bus A at different times. Each station transmits at a particular priority level. Starting time and priority level of the file transfers for all stations are shown in Table $1^{5}$. We assume that station 4 transmits a file with a length of 5,000 segments, the files transmitted by other stations are assumed to be significantly larger. We set the distance between adjacent stations to $\Delta=25$ slots $^{6}$. The total round-trip delay of the bus is therefore given by 150 slots. Once every round-trip delay we measure the number of segments that each station was able to transmit. The total observation period is set to 35,000 slots.

\footnotetext{
${ }^{3}$ We set Basis to the round-trip slot delay of the bus, i.e., the sum of the slot lengths of bus A and bus B [1].

${ }^{4}$ The simulations were implemented using the simulator for dual bus network protocols presented in [2]

${ }^{5}$ The time unit is one slot. The simulation starts at $t=0$.

${ }^{6}$ At a transmission rate of $155 \mathrm{Mb} / \mathrm{s}$ one slot corresponds to a distance of about $500 \mathrm{~m}$ and to a transmission delay of about $2.8 \mu \mathrm{s}$.
} 


\begin{tabular}{|c|c|c|}
\hline Station & $\begin{array}{c}\text { Start of } \\
\text { Transmission }\end{array}$ & $\begin{array}{c}\text { Priority } \\
\text { Level }\end{array}$ \\
\hline station 1 & $t=1,000$ & 1 \\
station 2 & $t=4,000$ & 2 \\
station 3 & $t=9,000$ & 2 \\
station 4 & $t=17,000$ & 3 \\
\hline
\end{tabular}

Table 1: Simulation Parameters.

Figure 4 shows the results of the simulation for the new protocol with Basis $=150$. Each point in Figure 4 gives the percentage of the bandwidth on bus A that is used by a station for transmission, i.e, the throughput of the station, in an interval of one round-trip delay. When station 1 starts transmission (at priority level 1) it seizes the entire bandwidth. As soon as station 2 with priority-2 traffic becomes active, it immediately pre-empts transmissions from station 1 . When station 3 begins transmitting with priority 2, it shares the bandwidth with station 2 such that both stations 2 and 3 obtain half of the total bandwidth. At $t=17,000$, station 4 with traffic at priority level 3 pre-empts any traffic with a lower priority. When all 5,000 segments of station 4 are transmitted, stations 2 and 3 again share the available bandwidth. Note how quickly the new protocol can adapt to changes in the network load.

For comparison, in Figure 5 we present a simulation of the same scenario with the priority scheme given in [8]. As mentioned in section 2.2, the scheme given in [8] is based on the bandwidth balancing mechanism. We use the default value for the bandwidth balancing modulus $(\beta=8)$. Figure 5 shows that each time the load of the network changes, it takes considerable time to adjust to the new network load. Because of the long convergence time, station 4 is not able to pre-empt the traffic from lower priority stations. This results in significantly higher transmission times for high priority traffic compared to the new protocol.

The advantages of the new protocol become even more apparent when the slot distance between the stations is increased. Increasing the slot distance corresponds to increasing the physical distance between stations, or equivalently, increasing the transmission speed of the network. We present the same simulation scenario in Table 1 for a dual bus network with a slot distance of $\Delta=50$ 
and $\Delta=100$ slots between two adjacent stations. Again we measure the throughput once every round-trip delay. For $\Delta=50$ slots we present simulation results for a total observation period of 50,000 slots. The results are shown in Figure 6 for the new priority scheme (with Basis $=300$ ) and in Figure 7 for the priority scheme from [8]. The new priority scheme is insensitive to doubling the slot distance between the stations, i.e., doubling the transmission rate of the bus. However, using the priority scheme from [8], the convergence time after load changes increases significantly compared to the previous simulation.

For $\Delta=100$ we choose an observation period of 65,000 slots. Again the priority scheme of our protocol shows to be effective as shown in Figure 8. In Figure 9 we show that the bandwidth balanced priority scheme [8] is almost ineffective. Although station 4 (with priority 3 ) becomes active at $t=17,000$, it achieves a non-zero throughput for the first time at about $t=40,000$.

In Table 2, we present the exact transmission time of the priority 3 file transmitted from station 4 for all simulation runs. The transmission time is the interval in slot times (see footnote 5 ) from the time station 4 becomes active (at $t=17,000$ ) until the last of its 5,000 segments is transmitted. The table clearly shows the superiority of our new protocol.

\begin{tabular}{|l|c|c|c|}
\hline & $\Delta=25$ & $\Delta=50$ & $\Delta=100$ \\
\hline New Protocol & 5,076 & 5,152 & 5,302 \\
\hline $\begin{array}{l}\text { Protocol in [8] } \\
\text { with } \beta=8\end{array}$ & 12,732 & 17,785 & 38,440 \\
\hline
\end{tabular}

Table 2: Transmission Delay for File from Station 3 (File Length $=5,000$ Segments). 


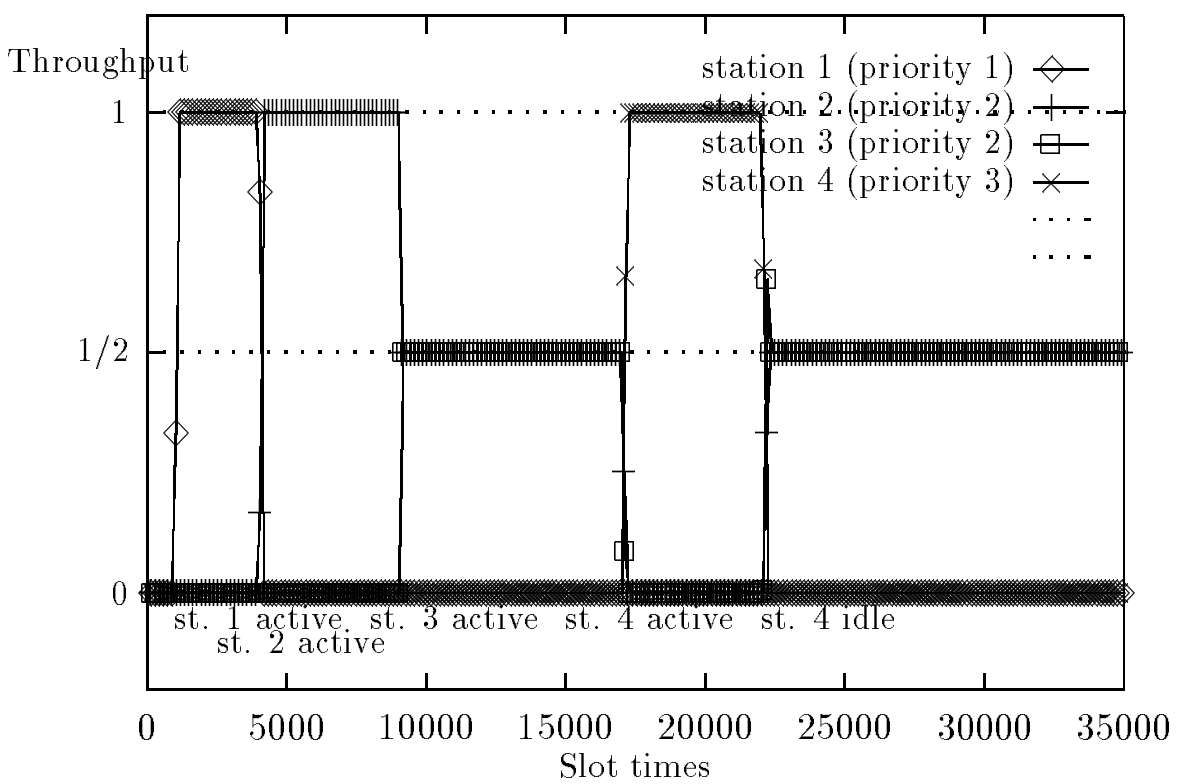

Figure 4: File Transfer with New Priority Scheme (Basis $=150$ ). 


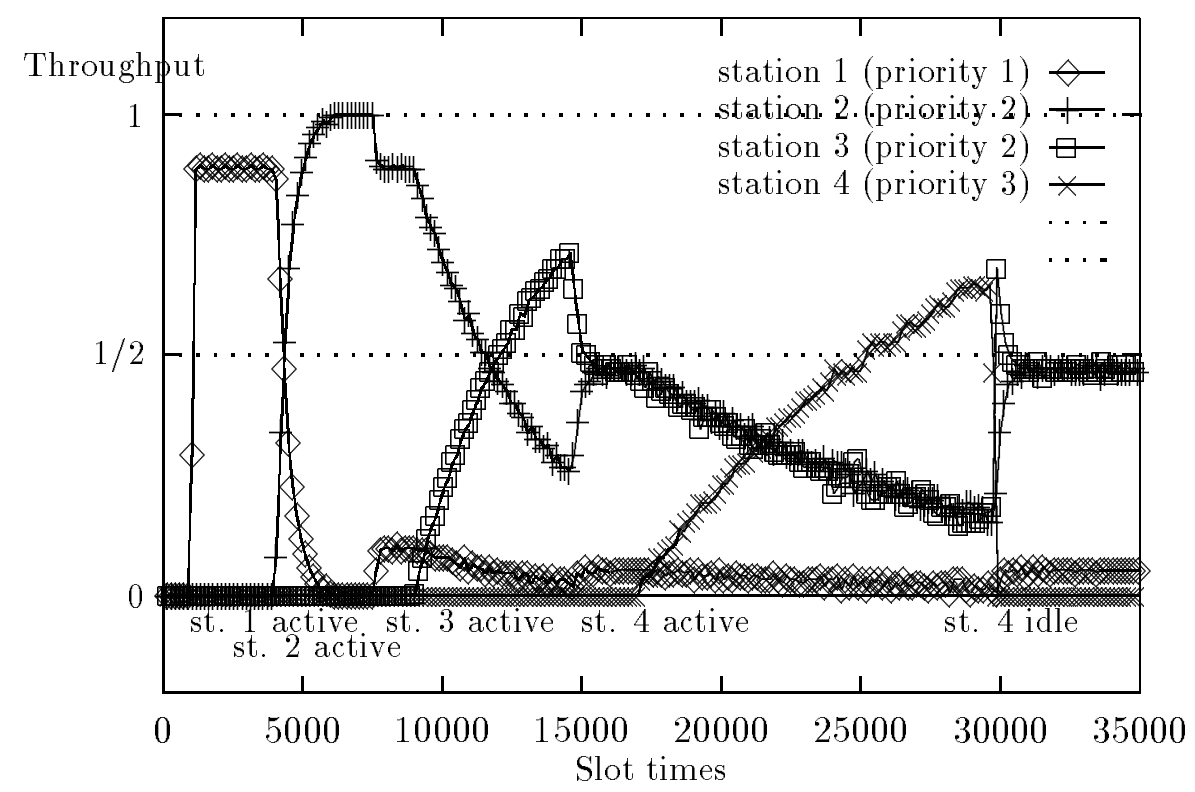

Figure 5: File Transfer with Priority Scheme in [7] $(\beta=8)$.

(Round-trip delay $=150$ slots) 


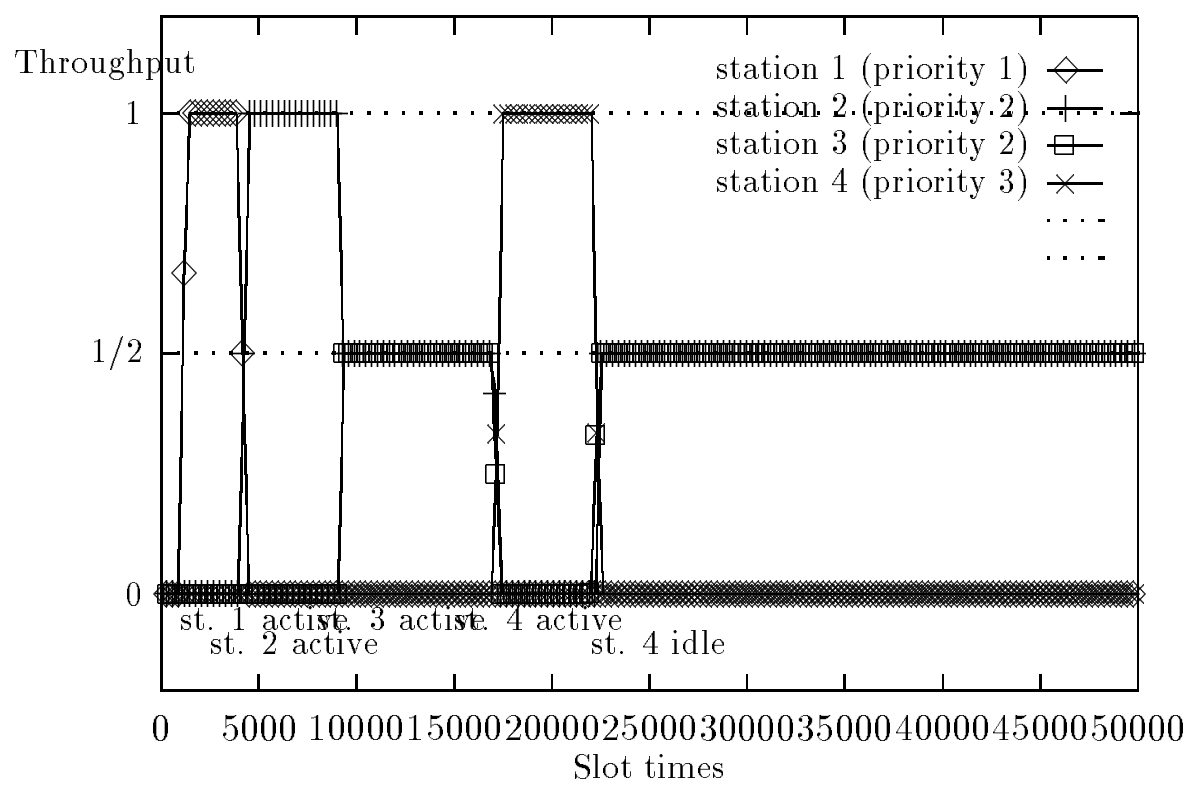

Figure 6: File Transfer with New Priority Scheme (Basis $=300$ ). 


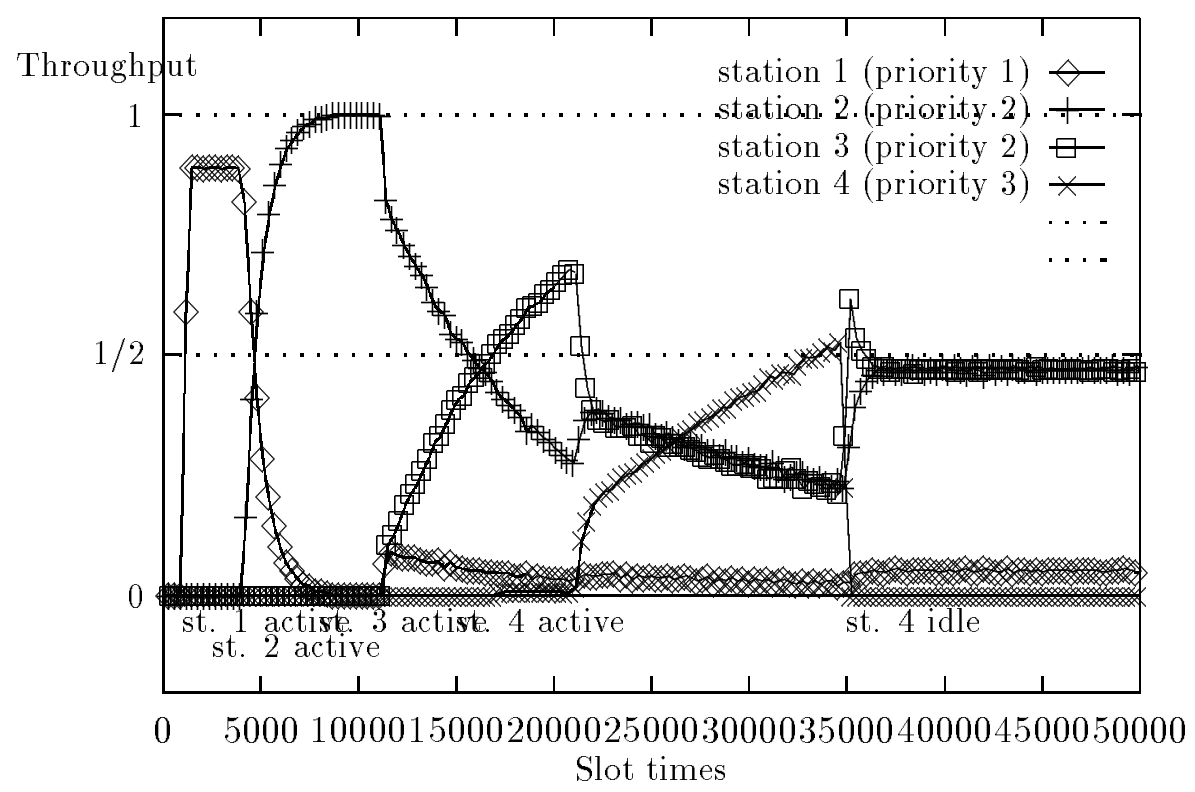

Figure 7: File Transfer with Priority Scheme in [7] $(\beta=8)$.

(Round-trip delay $=300$ slots) 


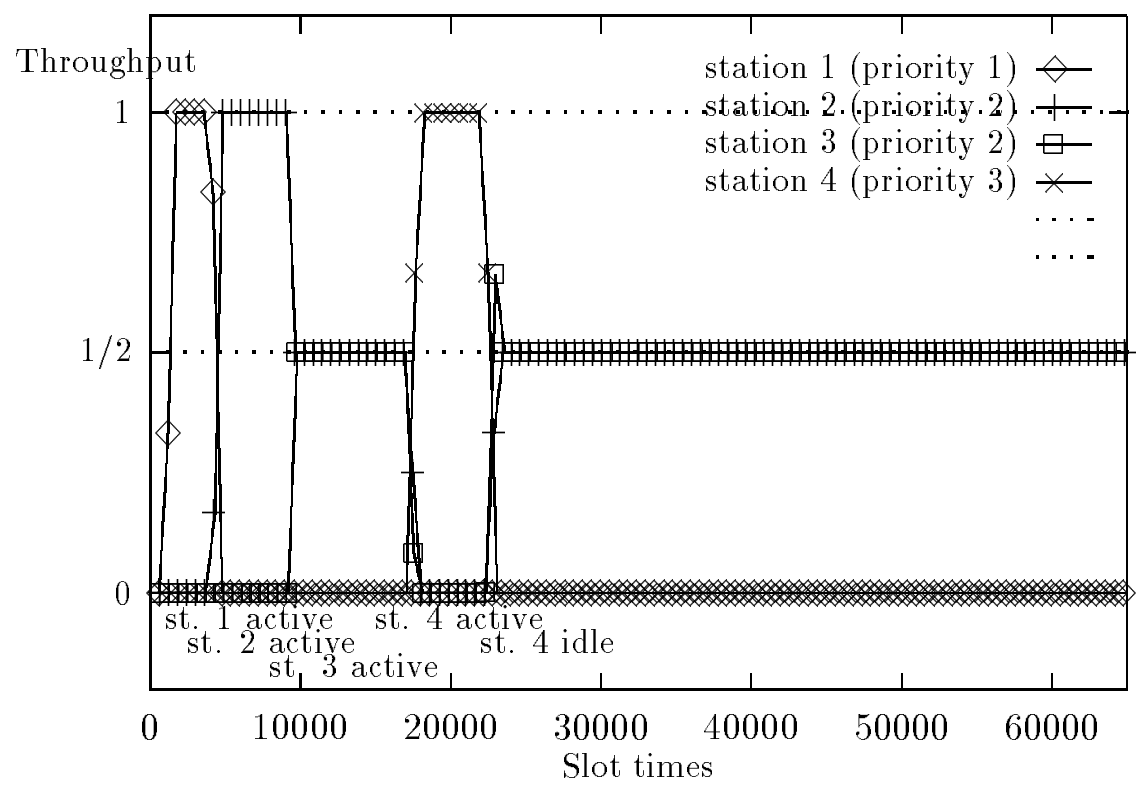

Figure 8: File Transfer with New Priority Scheme (Basis $=600$ ). 


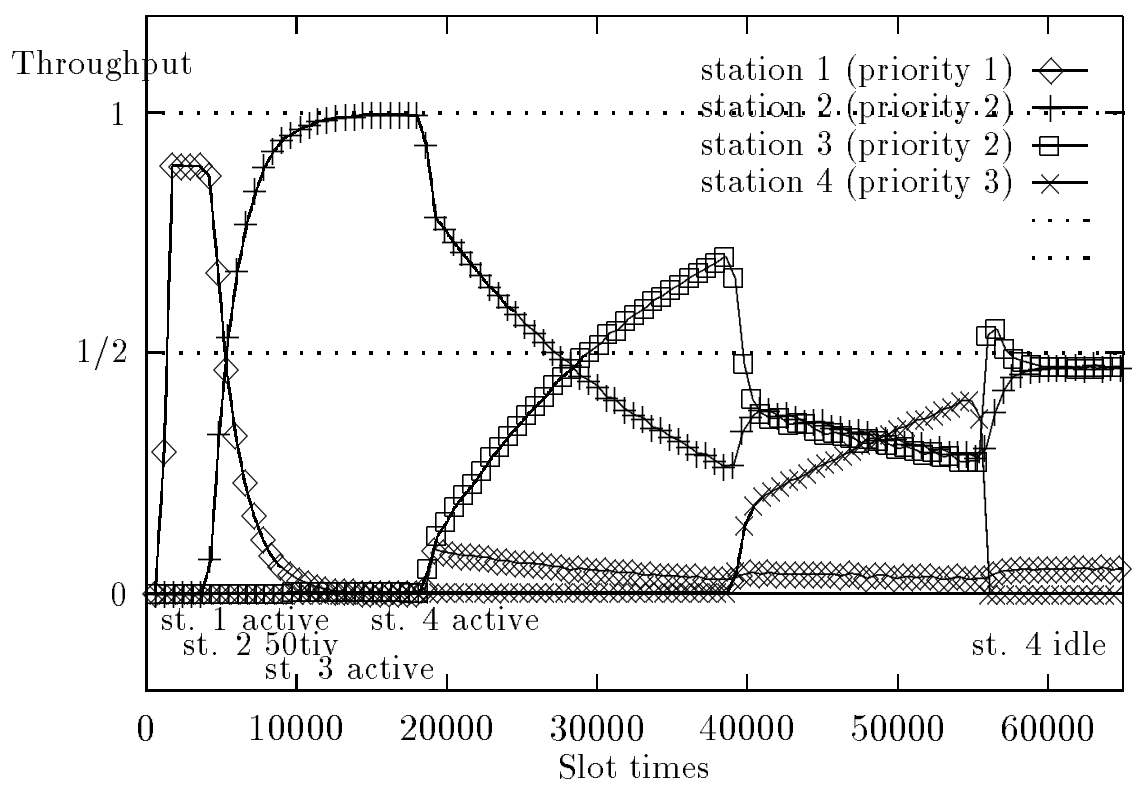

Figure 9: File Transfer with Priority Scheme in [7] $(\beta=8)$.

(Round-trip delay $=600$ slots) 


\section{Conclusions}

We provided a unified view on priority mechanisms for dual bus networks by formalizing properties of bandwidth allocation schemes. We showed deficiencies of existing protocols that support multiple priority traffic. We presented a new priority mechanism that does not waste bandwidth, provides a fair distribution of bandwidth within each priority level, and provides pre-emptive priorities. We proved the uniqueness of the priority mechanism. We introduced a media access protocol that is able to provide the unique priority mechanism. We showed that the new protocol achieves the implementation of pre-emptive priorities. The performance of the protocol was compared to an implementation of a priority mechanism that provides strong priorities and strong fairness (according to our terminology used in section 3). Our protocol adapts quicker adapt to changes

in the network load. We achieve a significantly lower transmission delay for high priority traffic compared to other priority schemes.

\section{References}

[1] I.F. Akyildiz, J. Liebeherr, and A.N. Tantawi. DQDB ${ }^{+/-}$- a Fair and Waste-Free Media Access Protocol for Dual Bus Metropolitan Networks. to appear in: IEEE Transactions on Communications.

[2] C. Bisdikian. DQDB Delay Analysis: The BWB Mechanism Case. In 4th IEEE Workshop on MANs, pages 5.1.1 - 5.1.28, Captiva Islands, FL, November 1990.

[3] C. Bisdikian and A. Tantawy. A Mechanism for Implementing Preemptive Priorities in DQDB Subnetworks. In Proc. ICC 1991, pages 1062-1067, Denver, CO, June 1991.

[4] R. Breault. Enhancement to the Bandwidth Balancing Mechanism. In Contribution No. 802.6-90/03 to the IEEE 802.6 Working Group, January 1990.

[5] V. Catania, L. Mazzola, A. Puliafito, and L. Vita. Throughput Analysis of DQDB in Overload Conditions. In Proc. ICC 1991, pages 741-747, Denver, CO, June 1991. 
[6] M. Conti, E. Gregori, and L. Lenzini. DQDB Media Access Control Protocol: Performance Evaluation and Unfairness Analysis. In 3rd IEEE Workshop on MANs, pages 375 - 408, San Diego, CA., March 1990.

[7] E. L. Hahne, A. K. Choudhury, and N. F. Maxemchuk. Improving the Fairness of Distributed Queue-Dual-Bus Networks. In Proc. INFOCOM'90, pages 175-184, San Francisco, CA, June 1990.

[8] E. L. Hahne and N. F. Maxemchuk. Fair Access of Multi-Priority Traffic to Distributed-Queue Dual-Bus Networks. In Proc. INFOCOM'91, pages 889 - 900, Bal Harbour, FL, April 1991.

[9] K. Han and I. Hyun. The Dynamic Bandwidth Balancing Mechanism for Improving DQDB Performance. In Proc. ICC 1991, pages 1345 - 1349, June 1991.

[10] IEEE. 802.6 Working Group, Proposed Standard: Distributed Queue Dual Bus (DQDB), Subnetwork of a Metropolitan Area Network (MAN), February 1990. P802.6/D12, Unapproved Draft.

[11] IEEE. Std 802.6-1990, IEEE Standards for Local and Metropolitan Area Networks: Distributed Queue Dual Bus (DQDB) of a Metropolitan Area Network (MAN), July 1991.

[12] D. J. Jeong, C. H. Choi, and W. S. Jeon. Fairness Improvement in DQ Protocols With Multiple Priorities. In Proc. ICC 1991, pages 1340 - 1344, June 1991.

[13] S. Y. Kim and K. J. Han. An Enhanced Bandwidth Balancing Mechanism of the DQDB MAN. In Proc. ICC 1991, pages 423 - 427, Denver, CO, June 1991.

[14] B. Mukherjee and C. Bisdikian. A Journey Through the DQDB Literature. Technical Report RC 17016, IBM Research Division, July 1991.

[15] M. Spratt. A Problem in the Multi-Priority Implementation of the Bandwidth Balancing Mechanism. In Contribution to the IEEE 802.6 Working Group, November 1989.

[16] M. Spratt. Implementing the Priorities in the Bandwidth Balancing Mechanism. In Contribution No. 802.6-90/05 to the IEEE 802.6 Working Group, January 1990. 
[17] M. P. Spratt. Allocation of Bandwidth in IEEE802.6 using Non-Unity Ratio Bandwidth Balancing. In Proc. ICC 1991, pages 729-735, Denver, CO, June 1991.

[18] H. R. van As. Major Performance Characteristics of the DQDB MAC Protocol. In Proc. SBT/IEEE International Telecommunications Symposium, pages 113 - 120, Rio de Janeiro, Brazil, September 1990.

[19] H. R. van As. Performance Evaluation of the Bandwidth Balancing in the DQDB MAC Protocol. In Proc. 8th Annual EFOC/LAN Conference, pages 231 - 239, June 1990.

[20] H. R. van As, J. W. Wong, and P. Zafiropoulo. Fairness, Priority and Predictability of the DQDB MAC Protocol under Heavy Load. In Proc. 1990 International Seminar on Digital Communication, pages 410 - 417, Zurich, Switzerland, March 1990. 


\section{A Proof of Theorem 1}

We first prove the existence and uniqueness of a solution for $\Omega^{*}$. Then we show that $\Omega^{*}$ is strongly fair, waste-free, and implements strong-priorities.

1. Existence of Solution for $\Omega^{*}$.

Since $\Omega^{*}$ is determined by a solution to the equation system of equations (5), (6) and (7), we construct such a solution. Denote $\hat{p}$ by:

$$
\hat{p}= \begin{cases}0 & \text { if }\|\Lambda\|<1 \\ \max _{p}\left\{\sum_{q=p}^{P}\left\|\Lambda_{q}\right\|>1\right\} & \text { otherwise }\end{cases}
$$

Without loss of generality we re-order the station indexes within priority level $\hat{p}$ such that $\lambda_{i \hat{p}} \leq \lambda_{j \hat{p}}$ if $i<j$. Note that in this case $i \in \mathbf{U}_{\hat{p}}$ implies $j \in \mathbf{U}_{\hat{p}}$, if $j<i$. A solution to $\Omega^{*}$ is then given by:

(a) $p<\hat{p}$ :

$$
\begin{aligned}
& \mathbf{U}_{p}=\left\{j \mid \lambda_{j p}=0\right\} \\
& \mathbf{O}_{p}=\left\{j \mid \lambda_{j p}>0\right\}
\end{aligned}
$$

Note that this implies that $\Omega_{i p}^{*}(\Lambda)=0$ for all $p<\hat{p}$ and all $i(1 \leq i \leq N)$.

(b) $p=\hat{p}$ :

$$
\begin{aligned}
& \mathbf{U}_{p}=\left\{j \mid \lambda_{j p} \leq \frac{1-\sum_{i=1}^{j} \lambda_{i p}-\sum_{k=1}^{N} \sum_{q=p+1}^{P} \lambda_{k q}}{N-j}\right\} \\
& \mathbf{O}_{p}=\mathcal{N} \backslash \mathbf{U}_{p}
\end{aligned}
$$

(c) $p>\hat{p}:$

$$
\begin{aligned}
& \mathbf{U}_{p}=\mathcal{N} \\
& \mathbf{O}_{p}=\emptyset
\end{aligned}
$$

2. Uniqueness of Solution for $\Omega^{*}$.

We assume the existence of a solution to equations (5), (6) and (7) that is different from the solution given by equations (13) - (19). Assume that the solution satisfies:

$$
\left(\exists p^{\prime}\right)\left(p^{\prime}<\hat{p}\right)(\exists i)(1 \leq i \leq N)\left(\lambda_{i p^{\prime}}>0 \rightarrow \Omega_{i p^{\prime}}^{*}(\Lambda)>0\right)
$$


Since $\sum_{q=\hat{p}}^{P}\left\|\Lambda_{q}\right\|>1$ (equations (13) and (19)) there exists a pair $(j, q)(1 \leq j \leq N, \hat{p}<q \leq$ $P$ ) such that $\lambda_{j q}>\Omega_{j q}(\Lambda)$. Because of equation (7), we obtain $\left|\mathbf{O}_{q}\right|>0$, and from equation (5) $\Omega_{i p^{\prime}}(\Lambda)=0$. But this contradicts the assumption.

Assume that there exists a solution to equations (5), (6) and (7) which satisfies:

$$
(\exists p)(p>\hat{p})\left(\left|\mathbf{O}_{p}\right|>0\right)
$$

Let $p^{\prime \prime}$ be the highest priority level $p$ with $p>\hat{p}$. For each $j \in \mathbf{O}_{p^{\prime \prime}}$ we have (equation (5)):

$$
\lambda_{j p^{\prime \prime}}>\frac{1-\sum_{q \geq p^{\prime \prime}, j \in \mathbf{U}_{q}} \lambda_{j q}}{\left|\mathbf{O}_{p^{\prime \prime}}\right|}
$$

Summing over all $j \in \mathbf{O}_{p^{\prime \prime}}$ we obtain:

$$
\begin{aligned}
\sum_{j \in \mathbf{O}_{p^{\prime \prime}}} \lambda_{j p^{\prime \prime}} & >1-\sum_{q \geq p^{\prime \prime}, j \in \mathbf{U}_{q}} \lambda_{j q} \\
& =1-\sum_{j \in \mathbf{U}_{p^{\prime \prime}}} \lambda_{j p^{\prime \prime}}-\sum_{j=1}^{N} \sum_{q=p^{\prime \prime}+1}^{P} \lambda_{j q}
\end{aligned}
$$

This is equivalent to:

$$
\sum_{p \geq p^{\prime \prime}}\left\|\Lambda_{p}\right\|>1
$$

But this contradicts equation (13).

Therefore, any solution to the equation system of equations (5), (6), and (7) must have $\mathbf{U}_{p}$ and $\mathbf{O}_{p}$ as given in equations (14) and (15) if $p<\tilde{p}$, and as given in equations (18) and (19) if $p>\tilde{p}$, respectively. Now, we investigate the case $p=\tilde{p}$. We show that any $\mathbf{U}_{\tilde{p}}$ and $\mathbf{O}_{\tilde{p}}$ that are part of a solution to equations (5), (6), and (7), are equivalent to $\mathbf{U}_{\tilde{p}}$ and $\mathbf{O}_{\tilde{p}}$ as given in equations (16) and (17).

Let $\tilde{k}$ be the highest index in $\mathbf{U}_{\tilde{p}}$ of equation (16). Assume a solution to equations (5), (6), and (7). Denote the underload and overload sets for priority $p$ of this solutions by $\overline{\mathbf{U}}_{p}$ and $\overline{\mathbf{O}}_{p}$, respectively. Let $k$ be the highest index in $\overline{\mathbf{U}}_{\tilde{p}}$. Per definition of $\mathbf{U}_{\tilde{p}}$ (equation (16)), it holds that $k \leq \tilde{k}$. On the other hand, since $(k+1) \in \overline{\mathbf{O}}_{\tilde{p}}$ :

$$
\begin{aligned}
\lambda_{k+1, \tilde{p}} & >\Omega_{k+1, \tilde{p}}^{*}(\Lambda) \\
& =\frac{1-\sum_{q \geq \tilde{p}, j \in \mathbf{U}_{q}} \lambda_{j q}}{\left|\overline{\mathbf{O}}_{\tilde{p}}\right|}
\end{aligned}
$$




$$
=\frac{1-\sum_{j=1}^{k} \lambda_{j \tilde{p}}-\sum_{q>\tilde{p}, j \in \mathbf{U}_{q}} \lambda_{j q}}{N-k}
$$

This is equivalent to:

$$
\lambda_{k+1, \tilde{p}}>\frac{1-\sum_{j=1}^{k+1} \lambda_{j \tilde{p}}-\sum_{q>\tilde{p}, j \in \mathbf{U}_{q}} \lambda_{j q}}{N-k-1}
$$

From equation (16), we obtain $k+1>\tilde{k}$, and as a consequence, $k=\tilde{k}$. Therefore, any solution to equations (5), (6), and (7) is given by equations (13) - (19).

3. $\Omega^{*}$ is strongly fair.

If $\left|\mathbf{O}_{p}\right|>0$ for some $p$ then the condition for strong fairness is satisfied with $\alpha_{q}^{*}=0$ for all $q<p$. On the other hand, if $\left|\mathbf{O}_{p}\right|=0$ for all $p>q, \alpha_{q}^{*}$ is given by:

$$
\alpha_{q}^{*}=\frac{1-\sum_{q \geq p, j \in \mathbf{U}_{q}} \lambda_{j q}}{|\mathbf{O}|_{q}} .
$$

4. $\Omega^{*}$ is waste-free.

If $\|\Lambda\|<1$, then $\mathbf{U}_{p}$ and $\mathbf{O}_{p}$ (for all $1 \leq p \leq P$ ) are given by:

$$
\begin{aligned}
& \mathbf{U}_{p}=\mathbf{N} \\
& \mathbf{O}_{p}=\emptyset
\end{aligned}
$$

Then, $\Omega_{i p}^{*}(\Lambda)=\min \left\{\lambda_{i p}, \infty\right\}=\lambda_{i p}$, and $\|\Gamma\|=\left\|\Omega^{*}(\Lambda)\right\|=\|\Lambda\|$.

For $\|\Lambda\|>1$ let $\tilde{p}$ be defined by:

$$
\tilde{p}=\max _{p}\left\{p|| \mathbf{O}_{p} \mid>0\right\} .
$$

Since $\Omega_{i p}^{*}(\Lambda)=0$ for all $p<\tilde{p}$, we obtain:

$$
\begin{aligned}
\sum_{i=1}^{N} \sum_{p=1}^{P} \Omega_{i p}^{*}(\Lambda) & =\sum_{i=1}^{N} \sum_{p=\tilde{p}}^{P} \min \left\{\lambda_{i p}, \frac{1-\sum_{q \geq p, j \in \mathbf{U}_{q}} \lambda_{j q}}{\left|\mathbf{O}_{p}\right|}\right\} \\
& =\sum_{i=1}^{N} \sum_{p=\tilde{p}+1}^{P} \lambda_{i p}+\sum_{i=1}^{N} \min \left\{\lambda_{i \tilde{p}}, \frac{1-\sum_{j=1}^{N} \sum_{q=\tilde{p}+1}^{P} \lambda_{j q}-\sum_{j \in \mathbf{U}_{\tilde{p}}} \lambda_{j \tilde{p}}}{\left|\mathbf{O}_{\tilde{p}}\right|}\right. \\
& =\sum_{i=1}^{N} \sum_{p=\tilde{p}+1}^{P} \lambda_{i p}+\sum_{i \in \mathbf{U}_{\tilde{p}}} \lambda_{i \tilde{p}}+\left|\mathbf{O}_{\tilde{p}}\right| \cdot \frac{1-\sum_{j=1}^{N} \sum_{q=\tilde{p}}^{P} \lambda_{j q}-\sum_{j \in \mathbf{U}_{\tilde{p}}} \lambda_{j \tilde{p}}}{\left|\mathbf{O}_{\tilde{p}}\right|} \\
& =1
\end{aligned}
$$


5. $\Omega^{*}$ implements strong priorities.

The condition for strong priorities holds since the calculation of $\Omega_{i p}^{*}$ in equations (5), (6), and

(7) is independent from arrival rates from lower priority levels $q<p$. 


\section{B Proof of Theorem 2}

For any pair $(i, p)(1 \leq i \leq N, 1 \leq p \leq P)$ we distinguish two cases:

1. $(\exists q>p)\left(\left|\mathbf{O}_{q}\right|>0\right)$

Since $\Omega^{*}$ has a unique solution we know from the construction of the solution in equations (13) - (19) that $\left|\mathbf{O}_{q}\right|>0$ implies for all $p<q$ :

$$
\begin{aligned}
& i \in \mathbf{U}_{p} \leftrightarrow \lambda_{i p}=0 \\
& i \in \mathbf{O}_{p} \leftrightarrow \lambda_{i p}>0
\end{aligned}
$$

Therefore, we just need to show that $\delta_{i p}=0$. If $\left|\mathbf{O}_{q}^{(>i)}\right|>0, \delta_{i p}=0$ is given by equation (19). For $\left|\mathbf{O}_{q}^{(>i)}\right|=0$ (but $\left|\mathbf{O}_{q}\right|>0$ ) we define $\tilde{j}$ and $\tilde{q}$ by:

$$
\begin{aligned}
& \tilde{q}=\max _{q}\left\{q>p \wedge\left|\mathbf{O}_{q}\right|>0 \wedge\left|\mathbf{O}_{q}^{(>i)}\right|=0\right\} \\
& \tilde{j}=\max _{j}\left\{j<i \wedge j \in \mathbf{O}_{q} \wedge j \notin \mathbf{O}_{q}^{(>i)}\right\}
\end{aligned}
$$

Then, we obtain in equation (5) for all $j \in \mathbf{O}_{\tilde{q}}$ :

$$
\Omega_{j \tilde{q}}^{*}(\Lambda)=\frac{1-\sum_{j \in \mathbf{U}_{\tilde{q}}} \lambda_{j \tilde{q}}}{\left|\mathbf{O}_{\tilde{q}}\right|}
$$

The sum of all $j \in \mathbf{O}_{\tilde{q}}$ yields:

$$
\sum_{j \in \mathbf{O}_{\tilde{q}}} \Omega_{j \tilde{q}}^{*}(\Lambda)=\left|\mathbf{O}_{\tilde{q}}\right| \cdot \frac{1-\sum_{j \in \mathbf{U}_{\tilde{q}}} \lambda_{j \tilde{q}}}{\left|\mathbf{O}_{\tilde{q}}\right|}
$$

Since $\left|\mathbf{O}_{\tilde{q}}^{(>i)}\right|=0, j \leq i$ holds for all elements $j \in \mathbf{O}_{\tilde{q}}$. Therefore, equation (45) gives:

$$
\sum_{j \leq i, j \in \mathbf{O}_{\tilde{q}}} \Omega_{j \tilde{q}}^{*}(\Lambda)+\sum_{j \in \mathbf{U}_{\tilde{q}}} \lambda_{j \tilde{q}}=1
$$

This is equivalent to:

$$
\sum_{j \leq i} \Omega_{j \tilde{q}}^{*}(\Lambda)+\sum_{j>i, j \in \mathbf{U}_{\tilde{q}}} \lambda_{j \tilde{q}}=1
$$

Since $\Omega^{*}$ is strongly fair, waste-free and has strong priorities, $\Omega_{j q}^{*}(\Lambda)=0$ for all $q<\tilde{q}$, and we obtain for $\delta_{p}$ :

$$
\delta_{p}=\frac{1-\sum_{j \leq i} \Omega_{j \tilde{q}}^{*}(\Lambda)-\sum_{j<i, j \in \mathbf{U}_{\tilde{q}}} \lambda_{j \tilde{q}}}{\left|\mathbf{O}_{p}^{(>i)}\right|+1}
$$


Inserting (47) into equation (48) gives:

$$
\delta_{p}=0
$$

2. $(\forall q>p)\left(\left|\mathbf{O}_{q}\right|=0\right)$

We consider $i \in \mathbf{O}_{p}$ and $i \in \mathbf{U}_{p}$ separately:

(a) $i \in \mathbf{O}_{p}$ :

Using equation (8), we can rewrite equation (5) as:

$$
\Omega_{i p}^{*}(\Lambda) \cdot\left(\left|\mathbf{O}_{p}^{(>i)}\right|+1\right)+\Omega_{i p}^{*}(\Lambda) \cdot\left(\left|\mathbf{O}_{p}\right|-\left|\mathbf{O}_{p}^{(>i)}\right|-1\right)=1-\sum_{q \geq p, j \in \mathbf{U}_{q}} \lambda_{j q}
$$

Because of equations (5) and $(7), \Omega_{j p}^{*}(\Lambda)=\Omega_{i p}^{*}(\Lambda)$ for all $j \in \mathbf{O}_{p}$, and we obtain:

$$
\Omega_{i p}^{*}(\Lambda) \cdot\left(\left|\mathbf{O}_{p}^{(>i)}\right|+1\right)=1-\sum_{q \geq p, j \in \mathbf{U}_{q}} \lambda_{j q}-\sum_{j \in \mathbf{O}_{p}, j<i} \Omega_{j p}^{*}(\Lambda)
$$

Recall that $\mathbf{O}_{q}=\emptyset$ for $q>p$. Since load and allocated bandwidth are equal for underloaded stations, i.e., $\Omega_{j r}^{*}(\Lambda)=\lambda_{j r}$ with $j \in \mathbf{U}_{r}$ and $1 \leq r \leq P$ (equation (6)), we have:

$$
\Omega_{i p}^{*}(\Lambda) \cdot\left(\left|\mathbf{O}_{p}^{(>i)}\right|+1\right)=1-\sum_{j>i, q \geq p, j \in \mathbf{U}_{q}} \lambda_{j q}-\sum_{q>p} \Omega_{i q}^{*}(\Lambda)-\sum_{j<i, q \geq p} \Omega_{j q}^{*}(\Lambda)
$$

and for all $i \in \mathbf{O}_{p}$ :

$$
\delta_{i p}=\Omega_{i p}^{*}(\Lambda)
$$

Using equation (7) finally yields equation (9).

(b) $i \in \mathbf{U}_{p}$ :

If $\left|\mathbf{O}_{p}^{(>i)}\right|=0$, then $j \in \mathbf{U}_{p}$ for $j>i$. On the other hand, we now that $j \in \mathbf{U}_{q}$ for $q>p$. We obtain:

$$
\sum_{q \geq p, j>i, j \in \mathbf{U}_{q}} \lambda_{j q}=\sum_{q \geq p, j>i, j \in \mathbf{U}_{q}} \Omega_{j q}^{*}(\Lambda)
$$

Let us assume that $\lambda_{i p}>\delta_{i p}$. Then, equation (9) reads:

$$
\lambda_{i p}>1-\sum_{q \geq p, j<i} \Omega_{j q}^{*}(\Lambda)-\sum_{q>p} \Omega_{i q}^{*}(\Lambda)-\sum_{q \geq p, j>i, j \in \mathbf{U}_{q}} \Omega_{j p}^{*}(\Lambda)
$$


Since $\lambda_{i p}=\Omega_{i p}^{*}(\Lambda)$ because $i \in \mathbf{U}_{p}$ (equation (6)), equation (55) contradicts Definition 1:

$$
\sum_{i=1}^{N} \sum_{p=1}^{P} \Omega_{i p}^{*}(\Lambda)=\|\Gamma\|<1
$$

If $\left|\mathbf{O}_{p}^{(>i)}\right|>0$, we select $k$ such that:

$$
k=\max _{j}\left\{j \in \mathbf{O}_{p}^{(>i)}\right\}
$$

From $k \in \mathbf{O}_{p}$, we obtain with equation (50):

$$
\Omega_{k p}^{*}(\Lambda)=\frac{1-\sum_{q \geq p, j>k, j \in \mathbf{U}_{q}} \lambda_{j q}-\sum_{q>p} \Omega_{k q}^{*}(\Lambda)-\sum_{q \geq p, j<k} \Omega_{j q}^{*}(\Lambda)}{\left|\mathbf{O}_{p}^{(>i)}\right|+1}
$$

Since $j \in \mathbf{U}_{p}$ for $i<j<k$, the following equations hold true:

$$
\begin{aligned}
\left|\mathbf{O}_{p}^{(>i)}\right| & =\left|\mathbf{O}_{p}^{(>k)}\right|+1 \\
\sum_{q \geq p, j>i, j \in \mathbf{U}_{q}} \lambda_{j q} & =\sum_{q \geq p, j>k, j \in \mathbf{U}_{q}} \lambda_{j q}+\sum_{q \geq p, i<j \leq k} \lambda_{j q} \\
& =\sum_{q \geq p, j>k, j \in \mathbf{U}_{q}} \lambda_{j q}+\sum_{q \geq p, i<j<k} \lambda_{j q}+\sum_{q>p} \lambda_{k q} \\
& =\sum_{q \geq p, j>k, j \in \mathbf{U}_{q}} \lambda_{j q}+\sum_{q \geq p, i<j<k} \Omega_{j q}^{*}(\Lambda)+\sum_{q>p} \Omega_{j q}^{*}(\Lambda) \\
\sum_{p \geq q, j<i} \Omega_{j q}^{*}(\Lambda) & =\sum_{p \geq q, j<k} \Omega_{j q}^{*}(\Lambda)-\sum_{p \geq q, i \leq j<k} \Omega_{j q}^{*}(\Lambda)
\end{aligned}
$$

Therefore equation (58) yields:

$$
\Omega_{k p}^{*}(\Lambda)=\frac{1-\sum_{q \geq p, j<k} \Omega_{j q}^{*}(\Lambda)-\sum_{q \geq p, j>i} \lambda_{j q}-\sum_{q \geq p} \Omega_{i p}^{*}(\Lambda)}{\left|\mathbf{O}_{p}^{(>i)}\right|}
$$

This can be re-written as :

$$
\Omega_{k p}^{*}(\Lambda) \cdot\left|\mathbf{O}_{p}^{(>i)}\right|+\Omega_{i p}^{*}(\Lambda)=1-\sum_{q \geq p, j<k} \Omega_{j q}^{*}(\Lambda)-\sum_{q \geq p, j>i} \lambda_{j q}-\sum_{q>p} \Omega_{i p}^{*}(\Lambda)
$$

Now, since $i \in \mathbf{U}_{p}$, we have $\lambda_{i p}=\Omega_{i p}^{*}(\Lambda)$. And since $i \in \mathbf{U}_{p}$ and $k \in \mathbf{O}_{p}$, we obtain $\Omega_{i p}^{*}(\Lambda)<\Omega_{k p}^{*}(\Lambda)$. Then equation (65) yields:

$$
\lambda_{i p}<\frac{\sum_{q \geq p, j<k} \Omega_{j q}^{*}(\Lambda)-\sum_{q \geq p, j>i} \lambda_{j q}-\sum_{q>p} \Omega_{i p}^{*}(\Lambda)}{\left|\mathbf{O}_{p}^{(>i)}\right|}
$$

